\title{
A Modified Boronic Acid (MBA) Disc Potentiation Assay for Detection of Extended Spectrum $\alpha$-Lactamase (ESBL) Producing Bacteria
}

\author{
Nazratan Naeem, Munawar Sultana, MAnwar Hossain* \\ ${ }^{1}$ Department of Microbiology, University of Dhaka, Dhaka 1000, Bangladesh
}

\begin{abstract}
In this study, a modified boronic acid (MBA) disc potentiation assay has been used to detect extended spectrum â-lactamase (ESBL) producing isolates from clinical liquid waste (CLW) in Bangladesh. The method is modified from the existing boronic acid (BA) disc potentiation assay in that, in our protocol only one type of antibiotic disc is required in comparison to the requirement of different types of antibiotic discs for the BA test. Clavulanic acid (CA) and Boronic acid (BA) were used to phenotypically identify ESBL and AmpC producers, respectively. A total of 181 multidrug-resistant (MDR) isolates were screened for ESBL production by both the conventional double disc diffusion synergy test (DDST) and the MBA disc potentiation test. Among these two methods, $\mathbf{3 8 \%}$ of the isolates showed positive ESBL phenotype in MBA test in comparison to the $24 \%$ of the isolates in DDST, hence showed higher efficiency. The MBA method described here is efficient, easy to perform, and cost effective to detect ESBL producing bacteria.
\end{abstract}

Keywords: Modified boronic acid disc potentiation assay, clinical liquid waste, ESBL, DDST

\section{Introduction}

ESBL stands for extended spectrum â-lactamase. They were first detected in $1979^{1}$. By nature, they are able to hydrolyze extended spectrum cephalosporin antibiotic with oxyimino side chain. ESBL enzymes confer resistance to several antibiotics such as cefotaxime, ceftriaxone, ceftazidime, aztreonam etc. ${ }^{2}$. AmpC are a group of chromosome encoded cephalosporinases that mediate resistance to cephalothin, cephazolin, cefoxitin, and â-lactamase inhibitor-â-lactam combinations ${ }^{3}$. ESBLs are widely distributed among gram positive and gram negative organism's, while AmpC are exclusively distributed among gram negative organisms, hence the differences among them are important to know ${ }^{3}$. Both these enzymes have acquired their importance in medical microbiology for both antimicrobial treatment and infection control in hospitals ${ }^{4}$. A few phenotypic detection methods for ESBL are in use now, to name some, double disc diffusion synergy test (DDST), National Committee for Clinical Laboratory Standards (NCCLS) screening test, NCCLS phenotypic confirmatory test (PCT) etc. Among them, DDST is one of the most popular methods for phenotypic identification of ESBL producing microorganisms. Due to an increase in the prevalence of multidrug-resistant ESBL microorganisms over the past few decades, many of the ESBL non-producers might appear as false positive ESBL producers. DDST might not be able to single-handedly identify them, because although this is an easy and reliable method for the detection of ESBL, yet the sensitivity of the test has been at question ${ }^{5}$. Therefore, a specific, sensitive, and easy to perform method is urgently needed to keep pace with the increasing number of ESBL producers. Based on the fact that boronic acid (BA) works as an inhibitor of AmpC production and clavulanic acid (CA) works as an inhibitor of ESBL production, quite recently, Boronic acid disc potentiation assay has been used in order to detect and discriminate between ESBL and AmpC producers $^{6}$. But this method requires specific antibiotics such as third generation cephalosporins and imipenem. However, we have tried to introduce a new method that is relatively inexpensive, and compared our result with the existing conventional approach. This method requires lesser types of antibiotic discs than other phenotypic characterization methods to detect ESBL producers. The prime objective of this study was to establish a novel approach beside conventional ones in order to detect ESBL producers phenotypically with less cost and labour intensity.

\section{Materials and Methods \\ ESBL producing isolates used in this study}

Three samples were collected from two renowned hospital sewage drains of Dhaka city: Dhaka Medical College Hospital (DMCH) emergency unit, DMCH outdoor unit, and Sir Salimullah Medical College Hospital (SSMCH) outflow; and antibiotic susceptibility test was performed using ampicillin, tetracycline, chloramphenicol, sulfamethoxazole, ceftazidime, cefotaxime, amikacin, ciprofloxacin, imipenem, aztreonam etc. Multidrug-resistant (MDR) isolates were selected for phenotypic detection of ESBL production.

${ }^{*}$ Corresponding author:

M Anwar Hossain, Department of Microbiology, University of Dhaka, Dhaka 1000, Bangladesh

Tel: +880 (02) 9661920-73, Ext 7735/7730; E-mail: hossaina@du.ac.bd 
Phenotypic detection of extended spectrum â-lactamase (ESBL) producing isolates

\section{Double disc diffusion synergy test (DDST)}

A total of 181 isolates from three samples were resistant to one or more â-lactam antibiotics and third generation cephalosporin groups of antibiotics in the previous multidrug resistance test. They were further detected for ESBL production by DDST phenotypic method ${ }^{7}$. Ceftazidime (CAZ 30 ìg), ceftriaxone (CRO 30 ìg), cefotaxime (CTX 30 Ìg) and amoxicillin/clavulanic acid (AMC 30 ìg) discs were used. Test inoculum was prepared by suspending 2-3 colonies on sterile nutrient broth.

The test inoculum, turbidity matched to 0.5 McFarland, was spread onto Mueller-Hinton agar (MHA) (Oxoid, UK) using a sterile cotton swab. A disc of augmenting (20 ìg amoxicillin + 10 ìg clavulanic acid) was placed on the surface of MHA; then discs of CRO, CAZ, and CTX were kept around it in such a way that each disc was at distance ranging between 15 and $20 \mathrm{~mm}$ from the augmenting disc (centre to centre). The plates were incubated at $37^{\circ} \mathrm{C}$ overnight. Distances between the discs were required to be suitably adjusted for each strain in order to accurately detect the synergy. The organisms were considered to be ESBL positive when the zone of inhibition around any of the expanded-spectrum cephalosporin discs showed a clear enhancement towards the augmenting disc.

\section{Modified boronic acid disc potentiation test}

This method is quite similar to that of the boronic acid disc potentiation test ${ }^{5}$, only difference is that, in our test we employed only amoxicillin (AML) (penicillin group of antibiotic) disc, whereas in BA test two cephalosporin group of antibiotics (cefotaxime and cefepime), and one monobactem antibiotic imipenem were used. All the other procedures followed in the modified test are exactly the same like that of the BA test. Finally, the present study was designed as follows: AML disc plus CA (AMC), AML plus BA(AML/BA), AML plus CA with BA(AML/ $\mathrm{CA} / \mathrm{BA})$, and AML disc alone in a single plate were placed on the lawn of the organism. CA was freshly prepared ( $2 \mathrm{~g} / \mathrm{l}$ of phosphate buffer saline at $\mathrm{pH} 6$ ) and 5 il was added to AML discs. Then 5 il of a 3-aminophenyl boronic acid stock solution (60 g/l) of dimethyl sulfoxide (DMSO) was added to AML and AMC discs. The final concentration of BA and CA on the discs was 300 ìg and 10 ìg respectively. The discs were allowed to dry for $60 \mathrm{~min}$ and used immediately. A lawn of test organism was made on the MuellerHinton agar (MHA) after adjusting the inoculum to $0.5 \mathrm{McF}$ arland unit and the discs were placed 15-20 mm distance apart (centre to centre) and incubated at $35^{\circ} \mathrm{C}$ for $18-24 \mathrm{~h}$ in ambient air. Ae" $5 \mathrm{~mm}$ increase in the zone diameter after addition of BA to the AML disc indicates a positive result for AmpC production, and a e" 5 $\mathrm{mm}$ increase in the zone diameter after addition of CA to the AML disc indicates a positive result for ESBL production.

\section{Boronic acid (BA) disc potentiation test}

Boronic acid disc potentiation test was also performed on the selected isolates (those were positive in DDST only) to differentiate the ESBL and AmpC â-lactamase producing isolates ${ }^{5}$. This test uses cefotaxime (CTX), CTX plus CA (CTX/ CA), CTX plus BA (CTX/BA) and CTX plus CA and BA (CTX/ CA/BA), cefepime (FEP), and FEP plus CA (FEP-CA) discs in a single plate with imipenem at the centre. Five microliter of the freshly prepared (2 $\mathrm{g} / \mathrm{l}$ of PBS at $\mathrm{pH}$ 6) CA was added to CTX, CAZ, and FEP discs. Then 5 il of a 3-aminophenyl BA (Sigma Aldrich, India) stock solution (60 g/l of dimethyl sulfoxide) was added to CTX and CTX plus CA discs. BA was added to the cefoxitin disc. A e" $5 \mathrm{~mm}$ increase in the zone of cefoxitin and in combination with BA indicates positivity for AmpC production. The final concentration of BA and CA on the discs was 300 ìg and 10 ìg, respectively. The discs were allowed to dry for $60 \mathrm{~min}$ and used immediately. A lawn of test organism was made on the Mueller-Hinton agar (MHA) after adjusting the inoculum to 0.5 McFarland unit and discs were placed and incubated at $35^{\circ} \mathrm{C}$ for $18-24 \mathrm{~h}$ in ambient air 5 . This individual test can differentiate among five different groups.

\section{Results}

Double disc diffusion synergy test

Among 181 multidrug resistant isolates from three CLW samples, 44 isolates (24\%, Figure 1) depicted the characteristic enhancement zone (Figure 2) towards the augmenting (AMC) amoxicillin/clavulanic acid disc. Among 44 DDST positive isolates, from DMCH emergency unit outflow 10 isolates (22.7\%) (ID: 1.1, 1.3, 1.4, 1.5, 1.12, 1.13, 1.15, 1.16, 1.18, 1.22) from DMCH outdoor unit effluents, 10 isolates (22.7\%) (ID: 2.1, 2.2, 2.4, 2.7, 2.10, 2.15 $2.18,2.24,2.29,2.57)$ and from SSMCH, 24 (54.54\%) (3.1, 3.6, 3.10, 3.13, 3.18, 3.24, 3.28, 3.32, 3.36, 3.39, 3.47, 3.49, 3.55, 3.56, 3.57, 3.67, 3.70, 3.72, 3.79, 3.84, 3.92, 3.98, 3.100, 3.104) isolates were DDST positive showing ESBL production.

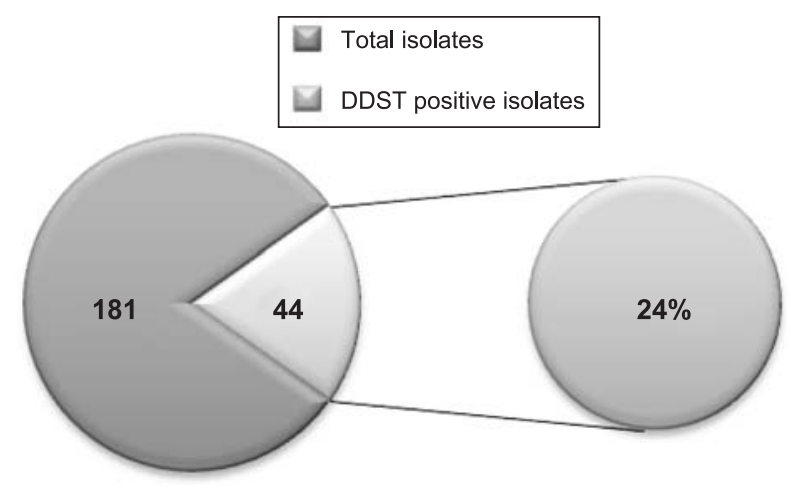

Figure 1: Number and percentage of double disc-diffusion synergy test positive isolates from three individual samples. 


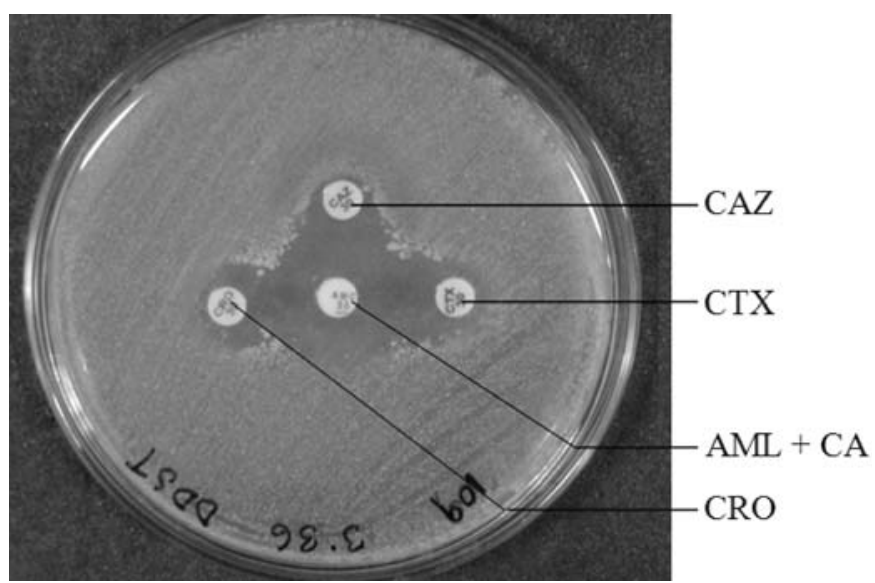

Figure 2: Double disc-diffusion test showing characteristics enhancement of zone of inhibition done with three third generation cephalosporin antibiotics along with an ESBL inhibitor (amoxicillin-clavulanic acid) in the middle. Isolate (ID: 3.36) has been shown here. CAZ = Ceftazidime; $C T X=$ Cefotaxime; $A M L=$ Amoxycillin; $C A=$ Clavulanic acid; $C R O=$ Ceftriaxone.

\section{Modified test for detection of ESBL}

A new method has been used to detect ESBL along with AmpC producers by using â-lactam antibiotic amoxicillin (AML), along with inhibitory agent boronic acid and clavulanic acid. A positive ESBL was indicative when zone diameter increased $>2 \mathrm{~mm}$ with clavulanic acid than amoxicillin alone. A e” $5 \mathrm{~mm}$ increase in the zone diameter of the AML alone and in combination with BA was considered positive for AmpC production (Figure 3).

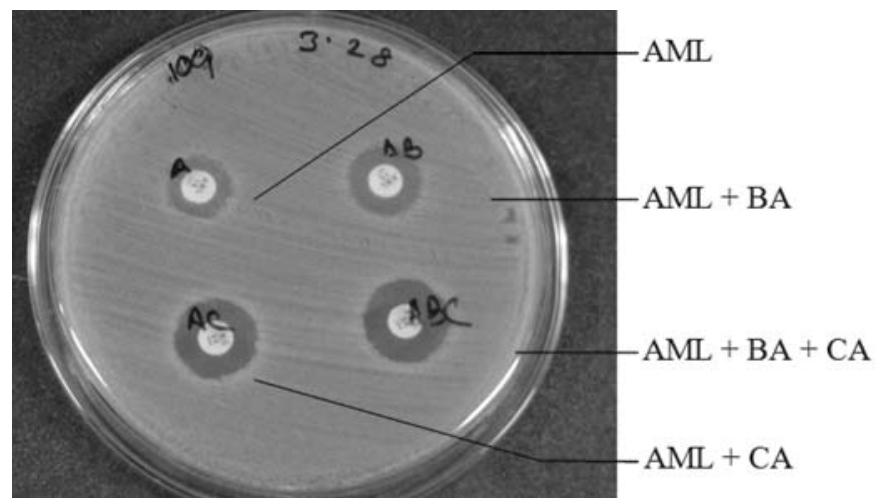

Figure 3: Modified boronic acid disc potentiation test of an isolate from SSMCH sewage sample. $A M L=$ Amoxycillin; $B A=$ Boronic Acid; CA= Clavulanic acid. Zone of inhibition increased with the addition of boronic acid, and clavulanic acid on amoxicillin disk individually.

To determine the efficiency of this newly designed test, all the 181 isolates were tested. Among them, 65 (38\%) were found to be ESBL or AmpC producers by this modified method, because addition of either clavulanic acid or boronic acid, or both, to the â-lactam antibiotic disc amoxicillin created a zone of inhibition around the bacterial lawn (Figure 4) showing a characteristic enhancement of zone diameter. Isolates from all three samples that were ESBL or AmpC, or both, producers belong to ID, from DMCH emergency unit: 1.4, 1.5, 1.6, 1.18 (6.15\%); from DMCH outdoor unit: 2.1, 2.4, 2.7, 2.10, 2.29 (7.69\%); and from SSMCH outflow: 3.1, 3.2, 3.3, 3.6, 3.7, 3.8, 3.16, 3.20-3.27, 3.29-3.32, 3.36, 3.37, 3.39-3.43, 3.47, 3.49-3.51, 3.55, 3.58-3.60, 3.62-3.67, 3.69-3.73, 3.88-3.89, 3.91, 3.92, 3.95, 3.97-3.98, 3.100, 3.101, 3.104, and 3.108 (86.15\%).

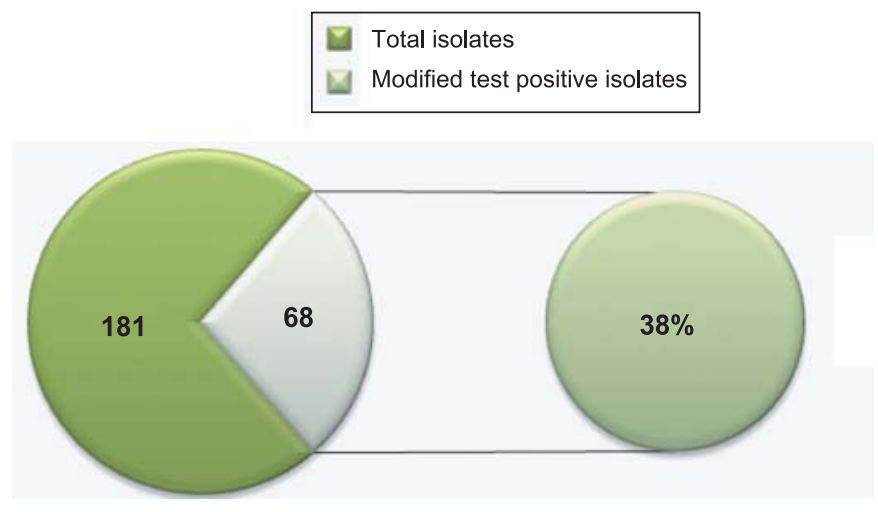

Figure 4: Percentage of isolates in modified boronic acid disc potentiation test.

\section{Boronic acid disc potentiation test}

Boronic acid disc potentiation test was done on 44 specific isolates that showed a positive result in double disc diffusion synergy test. According to boronic acid disc potentiation assay, isolates have been categorized into five different groups including pure ESBL and pure AmpC producers ${ }^{5}$ as mentioned in Figure 5. A total of 34 isolates among 44 DDST positive isolates (77\%) from three sewage samples of two different hospitals were found to be positive in BA test. The positive isolate IDs are as follows: From DMCH emergency unit 6 isolates - 1.3, 1.12, 1.13, 1.15, 1.18 and 1.22 (17.64\%); from DMCH outdoor unit 6 isolates - 2.1, 2.2, 2.15, 2.18, 2.24 and 2.29 (17.64\%); from SSMCH sewage 22 isolates 3.1, 3.6, 3.10, 3.13, 3.18, 3.24, 3.28, 3.36, 3.39, 3.47, 3.49, 3.55, 3.56, 3.57, 3.67, 3.70, 3.79, 3.84, 3.92, 3.98, 3.100 and 3.104 (64.7\%).

Production of ESBL enzyme is inhibited by CA, whereas AmpC production is inhibited by BA. Therefore, e" $5 \mathrm{~mm}$ increase in zone diameter with CTX alone and in combination with CA indicates pure ESBL producers, while e" $5 \mathrm{~mm}$ increase in zone diameter with CTX alone and in combination with BA indicates pure AmpC producers, respectively (Figure 5 b,c). Addition of both CA and BA solution to the CTX disc together resulted in an increase in the zone of inhibition, hence said be both pure AmpC and ESBL producers (Figure $5 \mathrm{~d}$ ). In the presence of imipenem disc in the middle, the zone of inhibition also increased (Figure 5 b) after adding BA to the CTX disc. Imipenem is used to detect inducible AmpC producers. A e” $5 \mathrm{~mm}$ increase in zone of 


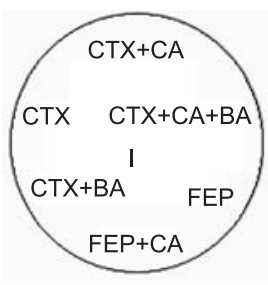

(a)

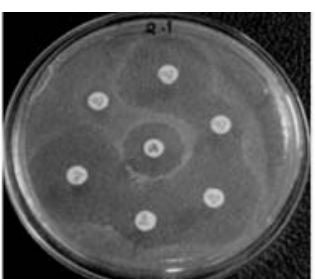

(b)

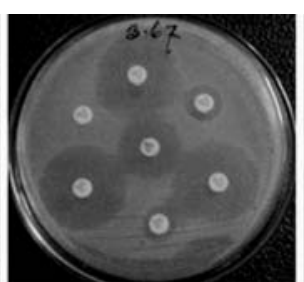

(c)

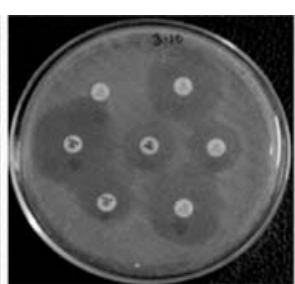

(d)

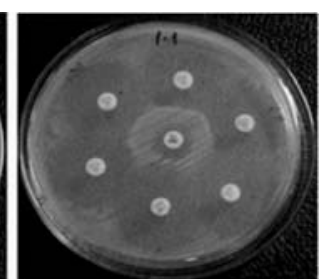

(e)

Figure 5: Boronic acid disc potentiation test showing (a) layout of the plates, (b) pure ESBL producer and inducible AmpC producer, (c) pure AmpC producers, (d) pure ESBL and AmpC producers, (e) harboring no enzyme. The test was performed in Muller Hinton agar which was swabbed with organism set at 0.5 McFarland unit. Addition of boronic acid and clavulanic acid to cefotaxime antibiotic disk individually increased the zone of inhibition.

inhibition diameter of FEP alone and in combination with CA indicates only ESBL, not AmpC production ${ }^{5}$.

Number of isolates showing ESBL or AmpC, or both, productivity in MBA test entailed larger number of isolates (44 isolates, $100 \%$ ) among the 44 DDST positive isolates than the phenotypically positive isolates in BA test (34 isolates, 77\%). Moreover, placement of seven antibiotic discs in one Petri-plate in the BA test in comparison to only 4 discs placed in the plate in the Modified test decreased the chance of fusion of zone of inhibition of the isolates.

\section{Discussion}

Elaborate phenotypic detection method of ESBL producers including conventional double disc diffusion synergy test (DDST), a modified boronic acid (MBA) disc potentiation test and boronic acid (BA) disc potentiation test has been included in this study.

By definition, ESBL enzymes are able to break down the characteristic â-lactam ring of the penicillin and cephalosporin group of antibiotic. Hence, adding boronic acid and/or clavulanic acid to the penicillin group of antibiotics should be able to detect ESBL producers with equal efficiency as the DDST. Therefore, we tried to establish a new method to detect the ESBL producers phenotypically, which we named as modified boronic acid (MBA) disc potentiation test. In BA test third generation cephalosporin group of antibiotics are employed to phenotypically identify and differentiate ESBL and AmpC producers, whereas in our modified method, we used only amoxicillin disc to interpret the same result. An increase in the zone of inhibition by e" $5 \mathrm{~mm}$ after addition of boronic acid to the amoxicillin disc was indication of AmpC production. Boronic acid (BA) derivatives were reported as reversible inhibitors of $\mathrm{AmpC}^{8-9}$, and an increase in the zone of inhibition by e" $5 \mathrm{~mm}$ after addition of clavulanic acid to the amoxicillin disc was indication of ESBL production by the isolates, since clavulanic acid is a potent inhibitor of ESBL enzymes ${ }^{10-11}$. By using this method, a total of 65 isolates among 181 showed an increase in the zone of inhibition after addition of boronic acid and/or clavulanic acid to the amoxicillin disc. However, 23 isolates among 181 from clinical liquid waste (CLW) showed positive phenotype for ESBL production in both DDST and MBA test. This method differs from DDST in that, an increase in the clear zone after adding boronic acid and clavulanic acid is measured, while on the other hand, DDST depicts the characteristic enhancement zone (Figure 2) towards the augmenting disc (amoxicillin/clavulanic acid). The new method needs less cost to detect the ESBL producers, since we need to use only 4 antibiotic discs to detect them (Figure 3). An easier comparison between zone of inhibition for amoxicillin disc alone and for amoxicillin/boronic acid and/or amoxicillin/clavulanic acid can be obtained by this method. This method was able to identify higher number (mentioned above) of ESBL producers than the conventional method.

Boronic acid disc potentiation test was performed on only 44 DDST positive isolates. Among them, 34 isolates were positive. These 34 isolates were also positive in our modified method. As a result, both the tests apparently seemed to be equally efficient in detecting ESBL producers phenotypically. What makes a difference between the tests is the simplicity of performing, inexpensiveness, and labour effectiveness of the modified method. Hence, the modified method for the identification of ESBLs is more efficient than other conventional approaches. Yet further molecular detection techniques are required to establish this method as a reliable identification method to detect and discriminate ESBL and AmpC producers together.

\section{Acknowledgement}

The author would like to acknowledge University Grants Commission (UGC) for monetary support.

\section{References}

1. Sanders CC and Sanders WE. 1979. Emergence of resistance to cefamandole: Possible role of cefoxitin-inducible beta-lactamases. Antimicrob Agents Chemother. 5(6): 792-797.

2. Emery CL and Weymouth LA. 1997. Detection and clinical significance of extended-spectrum beta-lactamases in a tertiary-care medical center. J Clin Microbiol. 35(8): 2061-2067.

3. Jacoby GA. 2009. AmpC â-lactamases. Clin Microbial Rev. 22(1): 161-182.

4. Drieux L, Brossier F, Sougakoff W and Jarlier V. 2008. Phenotypic detection of extended-spectrum beta-lactamase production in Enterobacteriaceae: Review and bench guide. Clin Microbiol Infect. 14: 90-103. 
5. Datta P, Thakur A, Mishra B, Gupta V. 2004. Prevalence of clinical strains resistant to various beta-lactams in a tertiary care hospital in India. Jpn J Infect Dis. 57(4): 146-149.

6. Shoorashetty RM, Nagarathnamma T and Prathibha J. 2011. Comparison of the boronic acid disc potentiation test and cefepimeclavulanic acid method for the detection of ESBL among AmpCproducing Enterobacteriaceae. Indian J Med Microbiol. 29: 297-301.

7. Jarlier V, Nicolas MH, Fournier G and Phillipon A. 1988. Extended broad-spectrum beta-lactamases conferring transferable resistance to newer beta-lactam agents in Enterobacteriaceae: Hospital prevalence and susceptibility patterns. Rev Infect Dis. 10: 868-878.
8. Beesely T, Gascoyne N, Knott-Hunziker V, Petursson S, Waley SG, Bengtake Jarunit B and Grundstrom T. 1982. The inhibition of class C â-lactamases by boronic acids. Biochem J. 209: 229-233.

9. Tondio D, Samuele C, Brian KS and Maria PC. 2010. Structural study of phenyl boronic acid derivatives as AmpC â-lactamase inhibitors. Bioorg Med Chem Lett. 20(11): 3416-3419.

10. Paterson DL and Bonomo RA. 2005 Extended-spectrum blactamases: A clinical update. Clin Microbiol Rev. 18: 657-686.

11. Bradford PA. 2001. Extended-spectrum â-lactamases in the $21^{\text {st }}$ century: Characterization, epidemiology, and detection of this important resistance threat. Clin Microbiol Rev. 14: 933-951. 